Review Article

\title{
HMGB 1 and Histones Play a Significant Role in Inducing Systemic Inflammation and Multiple Organ Dysfunctions in Severe Acute Pancreatitis
}

\author{
Runkuan Yang, ${ }^{1,2,3}$ Jyrki Tenhunen, ${ }^{1,4}$ and Tor Inge Tonnessen ${ }^{3,5}$ \\ ${ }^{1}$ Department of Intensive Care Medicine, Tampere University Hospital, University of Tampere, 10 Biokatu, 33014 Tampere, Finland \\ ${ }^{2}$ Department of Critical Care Medicine, University of Pittsburgh Medical School, 3550 Terrace Street, Pittsburgh, PA 15261, USA \\ ${ }^{3}$ Department of Emergencies and Critical Care, Oslo University Hospital, 4950 Nydalen, 0424 Oslo, Norway \\ ${ }^{4}$ Department of Surgical Science, Anesthesiology and Intensive Care Medicine, Uppsala University, 75185 Uppsala, Sweden \\ ${ }^{5}$ Institute of Clinical Medicine, University of Oslo, Blindern, 0316 Oslo, Norway
}

Correspondence should be addressed to Runkuan Yang; runkuanyang@gmail.com

Received 6 October 2016; Accepted 13 November 2016; Published 21 February 2017

Academic Editor: Jean-Marc Cavaillon

Copyright (C) 2017 Runkuan Yang et al. This is an open access article distributed under the Creative Commons Attribution License, which permits unrestricted use, distribution, and reproduction in any medium, provided the original work is properly cited.

\begin{abstract}
Severe acute pancreatitis (SAP) starts as a local inflammation of pancreatic tissue that induces the development of multiple extrapancreatic organs dysfunction; however, the underlying mechanisms are still not clear. Ischemia-reperfusion, circulating inflammatory cytokines, and possible bile cytokines significantly contribute to gut mucosal injury and intestinal bacterial translocation (BT) during SAP. Circulating HMGB1 level is significantly increased in SAP patients and HMGB1 is an important factor that mediates (at least partly) gut BT during SAP. Gut BT plays a critical role in triggering/inducing systemic inflammation/sepsis in critical illness, and profound systemic inflammatory response syndrome (SIRS) can lead to multiple organ dysfunction syndrome (MODS) during SAP, and systemic inflammation with multiorgan dysfunction is the cause of death in experimental SAP. Therefore, HMGB1 is an important factor that links gut BT and systemic inflammation. Furthermore, HMGB1 significantly contributes to multiple organ injuries. The SAP patients also have significantly increased circulating histones and cellfree DNAs levels, which can reflect the disease severity and contribute to multiple organ injuries in SAP. Hepatic Kupffer cells (KCs) are the predominant source of circulating inflammatory cytokines in SAP, and new evidence indicates that hepatocyte is another important source of circulating HMGB1 in SAP; therefore, treating the liver injury is important in SAP.
\end{abstract}

\section{Introduction}

Acute pancreatitis (AP) is a relatively common disease, its severe form is potentially fatal, and SAP is associated with high mortality, ranging from 15 to $40 \%$ [1-8]. AP starts as a local inflammation of pancreatic tissue that induces the development of multiple extrapancreatic organs dysfunction including acute lung injury [8-10], general endothelial barrier dysfunction, liver injury, and gut barrier dysfunction [2, 6,8 ]; systemic inflammation is thought to be the key link to multiple organ dysfunction (MOD) in SAP [11, 12] and gut $\mathrm{BT}$ plays a critical role in triggering/inducing systemic inflammation $[13,14]$; however, the underlying mechanism of BT in SAP is still poorly understood. The inflammatory cytokines play a crucial role in the pathogenesis of SAP $[6,7,15,16]$ and the late proinflammatory cytokine HMGB1 is particularly important in SAP $[17,18]$, because the circulating HMGB1 levels can reflect the disease severity and are potent in augmenting systemic inflammation $[17,18]$, and emerging evidence shows that HMGB1 is also an important factor that mediates $85 \%$ of the gut BT in acetaminophen hepatotoxicity [19]; therefore, it is possible that HMGB1 also mediates BT in SAP. Except HMGB1, new investigations show that extracellular histones and DNAs might also significantly contribute to multiple organ injury during SAP [20-23]. Because hepatic KCs are the predominant source of circulating inflammatory cytokines (including HMGB1) in SAP [8], and new evidence indicates that hepatocyte is another important source of 
circulating HMGB1 in SAP $[15,16]$; therefore, the inflamed liver is an important contributor to the circulating HMGB1 and the liver is a promising therapeutic target to prevent $\mathrm{BT}$ and systemic inflammation during SAP. This manuscript focuses on the new development in gut BT and provides evidences to show that HMGB1, histones, and DNAs might significantly contribute to systemic inflammation and multiple organ injury during SAP.

1.1. Ischemia-Reperfusion, Circulating Inflammatory Cytokines, and Possible Bile Cytokines Contribute to Gut Barrier Dysfunction in SAP. Gut barrier dysfunction is frequently seen in SAP $[6,11,24,25]$ and clarifying the underlying mechanisms is important because the intestine is the biggest reservoir of bacteria in the body and leakage of bacteria or microbial products, notably LPS, from the intestinal lumen into the systemic circulation, leads to initiation or amplification of a systemic inflammation and MOD [26].

Intestinal ischemia-reperfusion significantly contributes to small intestinal injury during SAP $[27,28]$ by inducing gut mucosal injury and affecting gut muscular layer to impair gut motility, which is frequently seen in SAP [14], and the reduced intestinal motility significantly contributes to disrupted intestinal microflora and BT [14].

The circulating inflammatory cytokines including TNF$\alpha$, IL-6, and HMGB1 also contribute to gut mucosal injury [29-32], among these inflammatory cytokines, extracellular HMGB1 triggers and sustains the inflammatory response by inducing cytokine release and by recruiting leucocytes [33]. However, HMGB1 undergoes extensive posttranslational modifications, in particular acetylation and oxidation, which significantly modulate the functions of HMGB1 $[33,34]$. High levels of serum HMGB1, in particular of the hyperacetylated and disulfide isoforms, are sensitive disease biomarkers [33], and SAP patients have significantly increased serum HMGB1 levels [35, 36], which can reflect the severity of gut barrier injury [35]. The circulating HMGB1 contributes to gut mucosal hyperpermeability and induces evident BT during hemorrhagic shock and reperfusion [37], and exogenous HMGB1 injection is able to induce gut hyperpermeability and BT in normal mice [29].

Except from the circulating HMGB1, bile HMGB1 might also significantly contribute to intestinal mucosa injury and induces evident BT in SAP because SAP patients have significantly increased circulating LPS levels [38], and LPS injection reduces $40 \%$ of bile flow in normal rats [31]; adequate bile is important to maintain gut epithelial tight junction and intestinal bacterial homeostasis [31] and decreased gut luminal bile volume not only impairs intestinal tight junction, but also changes intestinal bacterial homeostasis to facilitate BT [31]. In addition, LPS injection (to normal animals) markedly increases bile TNF- $\alpha$ and HMGB1 levels, and the endotoxemic bile can induce gut mucosal hyperpermeability and evident BT in normal mice [31], and neutralization of bile HMGB1 can reverse endotoxemic bile induced intestinal mucosal hyperpermeability and BT in normal mice [31], suggesting that bile HMGB1 is able to cause gut mucosal injury and intestinal BT.
1.2. HMGB1 Plays a Significant Role in Mediating BT in SAP. SAP induces significant small intestinal injury [27, 28], and the failure of gut barrier is associated with BT [27], which is evident in SAP $[6,24,25]$. SAP patients have significantly increased serum LPS level, which is likely derived from the gut lumen or from translocated intestinal bacteria [38]. 68.8\% of the SAP patients have bacteraemia and these bacteria have been tested as gut-derived opportunistic pathogens [24], which likely derive from small bowel rather than from the colon [25]. Therefore, it is important to identify the key factor that mediates intestinal BT in SAP. Gut bacteria can adhere to the injured intestinal mucosa that is necessary but not sufficient to induce gut BT in which HMGB1 and other unknown factors are also needed, because evidence shows that exogenous HMGB1 injection can induce gut mucosal injury and evident BT in normal mice [29], and HMGB1 also contributes to gut barrier dysfunction in rats with SAP [32]; blockade of HMGB1 reduces $85 \%$ of BT but does not significantly improve gut mucosal injury during acetaminophen hepatotoxicity [19]; similarly, ethyl pyruvate (EP), which is a HMGB1 inhibitor [39], reduces $80 \%$ of the gut $\mathrm{BT}$ but did not significantly decrease intestinal mucosal hyperpermeability in a lethal SAP animal model [6]; this BT-inhibition effect is associated with EP inhibiting $80 \%$ of the hepatic tissue HMGB1 release [15]. These evidences indicate that HMGB1 might mediate gut $\mathrm{BT}$ in SAP, and this hypothesis is confirmed by the following experiment: neutralization of HMGB1 decreased $70 \%$ of the gut BT (4252 $\pm 205 \mathrm{CFU} / \mathrm{g}$ for the sham antibody group versus $1235 \pm$ $41 \mathrm{CFU} / \mathrm{g}$ for the anti-HMGB1 group, results are mean \pm SEM, $n=6$ for each group, unpublished data) but did not reduce gut mucosal hyperpermeability in another commonly used murine SAP model (7 hourly $50 \mu \mathrm{g} / \mathrm{kg}$ intraperitoneal injections of cerulean and a single intraperitoneal injection of $4 \mathrm{mg} / \mathrm{kg}$ Escherichia coli lipopolysaccharide). These data indicate that intestinal mucosal injury is essential but not sufficient for gut BT during SAP; HMGB1 plays a significant role in mediating (at least partly) gut BT in experimental SAP and BT is likely an active "transcellular" procedure.

1.3. Gut BT Induces/Triggers Systemic Inflammation and Sepsis. Gut BT not only contributes to pancreatic infection [14, 25], but also induces/triggers systemic inflammation/sepsis in critical illness $[13,14]$, and the profound systemic inflammation can lead to MOD and mortality in SAP $[9,14,27$, 40]. This is one of the important underlying mechanisms that SAP frequently affects extrapancreatic organs $[3,6]$, and the incidence of MOD in SAP is not available but certainly higher than the $20-30 \%$ of mortality rate in SAP [3]. Systemic inflammation with multiorgan dysfunction is the cause of death in a murine ligation-induced SAP, and systemic inflammation and MOD can lead to the preponderance of mortality (75\%) in this lethal SAP model [11] while bile duct ligation does not have mortality [11]. Therefore, the gut is thought to act as the starter of SIRS [27] and HMGB1 is an important factor that links gut barrier dysfunction and MOD during SAP. 
1.4. The Liver Itself Plays an Important Role in BT in SAP. AP frequently affects the liver $[6,15]$ that plays an important role in BT during SAP by virtue of its unique structure and immune surveillance function $[14,41]$. The liver is the largest organ in the body, hepatocytes account for $70-80 \%$ of the hepatic cytoplasmic mass and nonparenchymal cells make up $20-30 \%$ of the hepatic cytoplasmic mass [41]. Among the nonparenchymal cells, KCs, sinusoidal endothelial cells, and the natural killer (NK) lymphocytes exert cellular defence functions for the whole body but also for the liver itself [41]. The liver has a large number of immune cells that can be rapidly expanded in response to infection or injury by recruiting leukocytes from the circulations [42]. SAP frequently injures the liver $[6,15,16,40]$ and impairs the phagocytic function $[14,40]$; the impaired host defence fails to clear regional lymph nodes and resultantly facilitates BT $[14,40]$.

1.5. HMGB1, Extracellular Histones, and DNAs Contribute to Multiple Organ Injuries. About 20-30\% of acute pancreatitis patients develop SAP in clinical practice; the mortality rate in SAP is $20-30 \%$ [3]; however, most of the SAP related mortality is not due to pancreatic injury itself; systemic inflammation with multiorgan dysfunction is the cause of death [11]. Early inflammatory cytokines are certainly involved in the pathogenesis of SAP; however, due to the narrow window time of the early cytokines in clinic, the clinical significance of the early inflammatory cytokines is limited. Attention should be focused on the late inflammatory cytokines such as HMGB1 and the newly recognized histones because these damage associated molecular patterns (DAMPs) have much wider window time to treat the patients and these DAMPs also significantly contribute to multiple organ injuries. The circulating HMGB1 level is significantly higher in SAP patients than in patients with mild pancreatitis [43]; the serum HMGB1 levels are higher in SAP patients with organ dysfunction and infection than in patients without organ dysfunction or infection [36]; the serum HMGB1 levels in nonsurvivors are higher than those in survivors [36]; serum HMGB1 levels are positively correlated with disease severity scores [36]. Pancreatic tissue HMGB1 levels are significantly increased in experimental SAP [44]; HMGB1 promotes the pathogenesis of pancreatitis $[39,45]$, and inhibiting HMGB1 therapy ameliorates the pancreatic injury [46]. HMGB1 contributes to liver injury in ischemiareperfusion [47]. Exogenous HMGB1 injection is able to induce liver injury in normal mice [29]. HMGB1 impairs hepatocyte regeneration during acetaminophen hepatotoxicity and blockade of HMGB1 improves hepatocyte regeneration in acetaminophen overdose-induced fatal liver injury [48]. Anti-HMGB1 treatment protects against APAP hepatotoxicity in rats [49]. HMGB1 also contributes to renal ischemiareperfusion injury [50], sepsis-induced kidney injury [51], and severe acute pancreatitis related kidney injury [52]. HMGB1 is also found to significantly contribute to hemorrhagic shock related acute lung injury (ALI) [53], hypoxiainduced ALI [54], and severe acute pancreatitis related ALI [55].
The extracellular histones are another group of important DAMPs molecules [56], which are toxic to host cells and elicit immunostimulatory effect that results in tissue damage and inflammation [56, 57]. The necrotic tissue/the dying cells release HMGB1 and histones as DAMPs [57]; therefore, circulating histones are significantly increased in both SAP patients and experimental animals with SAP [20-23] and the circulating histones levels reflect the disease severity in experimental AP [21].

The on-admission circulating nucleosome (containing DNA and histones) levels can predict organ dysfunction during the hospitalization of AP patients [20], suggesting that extracellular histones might contribute to multiple organ dysfunctions in SAP. Reduced pancreatic injury is associated with decreased histones 3 and histone 4 levels in the pancreas in response to taurocholate challenge [23], suggesting that histones are associated with pancreatic injury during SAP. The extracellular histones kill endothelial cells and are one of the major mediators of death in sepsis [58]. Extracellular histones contribute to two different acute fatal liver injury models [57]; TLR2 and TLR4 are the major receptors for extracellular histone mediated sterile inflammation, tissue injury, and organ failure in acute liver failure (ALF) [57]; neutralization of histones can ameliorate these two different fatal liver injury models in mice [57]. Extracellular histones can induce microvascular endothelial injury and the TLR2/4mediated inflammation can lead to acute tubular necrosis in acute kidney injury (AKI) $[59,60]$. Extracellular histones injure endothelial cells causing microvascular thrombosis and hemorrhage in acute lung injury (ALI) [61]. Histones also contribute to stroke in acute brain injury, and blockade of histones can reduce infarct size [62]. Histone $\mathrm{H} 4$ and increased circulating neutrophil extracellular traps (NETs) activate platelets, which trigger platelet aggregation and clotting that result in microvascular thrombosis and vascular and parenchymal injury in sepsis $[57,58,60]$. Therefore, extracellular histones contribute to the microvascular complications of sepsis, small vessel vasculitis, acute fatal liver injury, acute kidney, and acute lung injuries [58, 60].

Cell-free plasma DNA is significantly increased in SAP patients $[20,22]$ and in experimental animal with SAP [22]. Damaged cells from SAP can release nucleosomes, which contain DNA and histones, into the circulation to promote inflammation, and the circulating nucleosomes levels can predict AP patients who present no clinical signs of organ dysfunction on admission and are bound to develop organ dysfunction during hospitalization [20], suggesting that DNA/or histones likely play a significant role in the development of organ dysfunction during SAP. Neutrophils play an active role in the development of AP [22]. Except the secretion of antimicrobial compounds, activated neutrophils eliminate invading microorganisms by expelling nuclear DNA and histones to form extracellular web-like structures called neutrophil extracellular traps (NETs) [22]. NETs form in the pancreas in a murine SAP model; addition of NETs and histones to the acinar cells induces trypsin formation and activates the signal transducer and transcription [22]. NETs are able to activate platelets leading to thrombosis and the major contributor to this process is histone 4 [52]. NETs 
contribute to organ dysfunction in patients with infectious diseases and regulate organ inflammation and injury in mice with AP [22]. SAP patients have increased plasma levels of NETs [22]. Administration of DNase 1 (to deplete DNA and NETs) decreases circulating HMGB1 level and reduces neutrophil infiltration and tissue damage in the inflamed pancreas and lung [22], suggesting that DNA also significantly contributes to the development of SAP.

1.6. The Inflamed Liver Is an Important Source of Circulating HMGB1 and Has an Impaired Capacity to Clear Histones in SAP. Proinflammatory mediators are thought to play a crucial role in the pathogenesis of SAP $[6,8,15,16$, $63,64]$. The amount of cytokines released from the liver represents about $50 \%$ of the total cytokine content in the body [64], suggesting that the liver is the major contributor of the circulating cytokines. KCs are the largest number of tissue resident macrophages in the body and a predominant source of circulating inflammatory cytokines in SAP [8], and KCs play a major role in amplifying systemic inflammation during AP [11, 12, 64-68]. Except KCs, emerging evidence is showing that hepatocyte is another important source of circulating HMGB1 in two different experimental SAP models $[15,16]$ : especially in this triple-hit lethal SAP model, the inflamed liver releases nearly $90 \%$ of the hepatic tissue HMGB1 (detected by liver tissue whole cell lysis) [15]. EP is a potent HMGB1 inhibitor that ameliorates SAP via the reduced serum HMGB1 level [17], which is potent in augmenting SIRS in SAP $[17,18]$. EP is also found to ameliorate intestinal barrier dysfunction by reducing the ileal mucosal HMGB1 expression [32]. EP not only inhibits LPS-stimulated macrophages to release TNF- $\alpha$ and HMGB1 [69], but also inhibits the inflamed liver to release both early (TNF- $\alpha$, IL6) and late (HMGB1) inflammatory mediators during SAP $[15,16]$ and resultantly ameliorates the SAP related multiple distant organs injuries (including the liver injury) [6, 15-18]. These evidences indicate that both KCs and the hepatocytes are an important source of circulating HMGB1 in SAP, and the inflamed liver might play a critical role in the translation of pancreatic injury into systemic inflammation and MOD. In addition, the liver is an important organ to rapidly clear extracellular histones [21]; however, SAP can induce severe liver injury and impair its capacity of clearing histones, and this can lead to the increased circulating histones levels, which can significantly contribute multiple organ injury as described above. Therefore, the liver could be a promising therapeutic target to treat SAP.

\section{Conclusions}

Increased circulating HMGB1, histones, and cell-free DNAs levels in SAP patients might play a significant role in contributing to systemic inflammation and multiple organ injury during SAP. HMGB1 is an important factor that might link gut BT and systemic inflammation in SAP. The inflamed liver is an important source of circulating HMGB1 and treating the liver injury is important in SAP.

\section{Competing Interests}

The authors declare that they have no competing interests.

\section{Acknowledgments}

This investigation was supported by South-Eastern Norway Regional Health Authority, Grant no. 2013121.

\section{References}

[1] M. Yousaf, K. McCallion, and T. Diamond, "Management of severe acute pancreatitis," British Journal of Surgery, vol. 90, no. 4, pp. 407-420, 2003.

[2] R. Hegazi, A. Raina, T. Graham et al., "Early jejunal feeding initiation and clinical outcomes in patients with severe acute pancreatitis," Journal of Parenteral and Enteral Nutrition, vol. 35, no. 1, pp. 91-96, 2011.

[3] Z.-W. Yang, X.-X. Meng, and P. Xu, "Central role of neutrophil in the pathogenesis of severe acute pancreatitis," Journal of Cellular and Molecular Medicine, vol. 19, no. 11, pp. 2513-2520, 2015.

[4] S. Isaji, T. Takada, Y. Kawarada et al., "JPN Guidelines for the management of acute pancreatitis: surgical management," Journal of Hepato-Biliary-Pancreatic Surgery, vol. 13, no. 1, pp. 48-55, 2006.

[5] S. L. Triester and K. V. Kowdley, "Prognostic factors in acute pancreatitis," Journal of Clinical Gastroenterology, vol. 34, no. 2, pp. 167-176, 2002.

[6] R. Yang, T. Uchiyama, S. M. Alber et al., "Ethyl pyruvate ameliorates distant organ injury in a murine model of acute necrotizing pancreatitis," Critical Care Medicine, vol. 32, no. 7, pp. 1453-1459, 2004.

[7] J. Escobar, J. Pereda, A. Arduini et al., "Role of redox signaling, protein phosphatases and histone acetylation in the inflammatory cascade in acute pancreatitis: therapeutic implications," Inflammation and Allergy-Drug Targets, vol. 9, no. 2, pp. 97108,2010

[8] H. Akbarshahi, A. H. Rosendahl, G. Westergren-Thorsson, and R. Andersson, "Acute lung injury in acute pancreatitis-a waiting the big leap," Respiratory Medicine, vol. 106, no. 9, pp. 1199-1210, 2012.

[9] D. Closa, L. Sabater, L. Fernández-Cruz, N. Prats, E. Gelpí, and J. Roselló-Catafau, "Activation of alveolar macrophages in lung injury associated with experimental acute pancreatitis is mediated by the liver," Annals of Surgery, vol. 229, no. 2, pp. 230236, 1999.

[10] D. Closa, M. Bardaji, G. Hotter et al., "Hepatic involvement in pancreatitis-induced lung damage," American Journal of Physiology-Gastrointestinal and Liver Physiology, vol. 270, no. 1, pp. G6-G13, 1996.

[11] Z. Yuan, D. K. Meyerholz, E. C. Twait, D. Kempuraj, D. E. Williard, and I. Samuel, "Systemic inflammation with multiorgan dysfunction is the cause of death in murine ligationinduced acute pancreatitis," Journal of Gastrointestinal Surgery, vol. 15, no. 10, pp. 1670-1678, 2011.

[12] E. Puy-Folch, "Importance of the liver in systemic complications associated with acute pancreatitis: the role of Kupffer cells," Journal of Pathology, vol. 211, no. 4, pp. 383-388, 2007.

[13] M. Y. Z. Fanous, A. J. Phillips, and J. A. Windsor, "Mesenteric lymph: the bridge to future management of critical illness," Journal of the Pancreas, vol. 8, no. 4, pp. 374-399, 2007. 
[14] N. Runkel, "Bacterial translocation in acute pancreatitis," Digestive Surgery, vol. 13, no. 4-5, pp. 269-272, 1996.

[15] R. Yang, A. L. Shaufl, M. E. Killeen, and M. P. Fink, "Ethyl pyruvate ameliorates liver injury secondary to severe acute pancreatitis," Journal of Surgical Research, vol. 153, no. 2, pp. 302-309, 2009.

[16] Z.-G. Luan, H. Zhang, X.-C. Ma, C. Zhang, and R.-X. Guo, "Therapeutic treatment with ethyl pyruvate attenuates the severity of liver injury in rats with severe acute pancreatitis," Pancreas, vol. 41, no. 5, pp. 729-737, 2012.

[17] Z.-Y. Yang, Y. Ling, T. Yin et al., "Delayed ethyl pyruvate therapy attenuates experimental severe acute pancreatitis via reduced serum high mobility group box 1 levels in rats," World Journal of Gastroenterology, vol. 14, no. 28, pp. 4546-4550, 2008.

[18] B.-Q. Cheng, C.-T. Liu, W.-J. Li et al., "Ethyl pyruvate improves survival and ameliorates distant organ injury in rats with severe acute pancreatitis," Pancreas, vol. 35, no. 3, pp. 256-261, 2007.

[19] R. Yang, X. Zou, J. Tenhunen et al., "HMGB1 neutralization is associated with bacterial translocation during acetaminophen hepatotoxicity," BMC Gastroenterology, vol. 14, no. 1, article 66, 2014.

[20] A. K. Penttilä, A. Rouhiainen, L. Kylänpää et al., "Circulating nucleosomes as predictive markers of severe acute pancreatitis," Journal of Intensive Care, vol. 4, article 14, 2016.

[21] X. Ou, Z. Cheng, T. Liu et al., "Circulating histone levels reflect disease severity in animal models of acute pancreatitis," Pancreas, vol. 44, no. 7, pp. 1089-1095, 2015.

[22] M. Merza, H. Hartman, M. Rahman et al., "Neutrophil extracellular traps induce trypsin activation, inflammation, and tissue damage in mice with severe acute pancreatitis," Gastroenterology, vol. 149, no. 7, pp. 1920-1931e8, 2015.

[23] M. Merza, M. Rahman, S. Zhang et al., "Human thrombinderived host defense peptides inhibit neutrophil recruitment and tissue injury in severe acute pancreatitis," American Journal of Physiology-Gastrointestinal and Liver Physiology, vol. 307, no. 9, pp. G914-G921, 2014.

[24] Q. Li, C. Wang, C. Tang, Q. He, N. Li, and J. Li, "Bacteremia in patients with acute pancreatitis as revealed by $16 \mathrm{~S}$ ribosomal RNA gene-based techniques," Critical Care Medicine, vol. 41, no. 8, pp. 1938-1950, 2013.

[25] S. Fritz, T. Hackert, W. Hartwig et al., "Bacterial translocation and infected pancreatic necrosis in acute necrotizing pancreatitis derives from small bowel rather than from colon," American Journal of Surgery, vol. 200, no. 1, pp. 111-117, 2010.

[26] M. P. Fink, "Leaky gut hypothesis: a historical perspective," Critical Care Medicine, vol. 18, no. 5, pp. 579-580, 1990.

[27] G. Capurso, G. Zerboni, M. Signoretti et al., "Role of the gut barrier in acute pancreatitis," Journal of Clinical Gastroenterology, vol. 46, pp. S46-S51, 2012.

[28] Z.-Z. Guo, P. Wang, Z.-H. Yi, Z.-Y. Huang, and C.-W. Tang, "The crosstalk between gut inflammation and gastrointestinal disorders during acute pancreatitis," Current Pharmaceutical Design, vol. 20, no. 7, pp. 1051-1062, 2014.

[29] P. L. Sappington, R. Yang, H. Yang, K. J. Tracey, R. L. Delude, and M. P. Fink, "HMGB1 B box increases the permeability of Caco-2 enterocytic monolayers and impairs intestinal barrier function in mice," Gastroenterology, vol. 123, no. 3, pp. 790-802, 2002.

[30] P. L. Sappington, X. Han, R. Yang, R. L. Delude, and M. P. Fink, "Ethyl pyruvate ameliorates intestinal epithelial barrier dysfunction in endotoxemic mice and immunostimulated caco2 enterocytic monolayers," Journal of Pharmacology and Experimental Therapeutics, vol. 304, no. 1, pp. 464-476, 2003.
[31] R. Yang, K. Miki, N. Oksala et al., "Bile high-mobility group box 1 contributes to gut barrier dysfunction in experimental endotoxemia," American Journal of Physiology-Regulatory Integrative and Comparative Physiology, vol. 297, no. 2, pp. R362-R369, 2009.

[32] Z.-G. Luan, H. Zhang, X.-C. Ma, C. Zhang, and R.-X. Guo, "Role of high-mobility group box 1 protein in the pathogenesis of intestinal barrier injury in rats with severe acute pancreatitis," Pancreas, vol. 39, no. 2, pp. 216-223, 2010.

[33] E. Venereau, F. De Leo, R. Mezzapelle, G. Careccia, G. Musco, and M. E. Bianchi, "HMGB1 as biomarker and drug target," Pharmacological Research, vol. 111, pp. 534-544, 2016.

[34] U. Andersson, D. J. Antoine, and K. J. Tracey, "The functions of HMGB1 depend on molecular localization and posttranslational modifications," Journal of Internal Medicine, vol. 276, no. 5, pp. 420-424, 2014.

[35] G.-F. Xu, M. Guo, Z.-Q. Tian, G.-Z. Wu, X.-P. Zou, and W.J. Zhang, "Increased of serum high-mobility group box chromosomal protein 1 correlated with intestinal mucosal barrier injury in patients with severe acute pancreatitis," World Journal of Emergency Surgery, vol. 9, article 61, 2014.

[36] T. Yasuda, T. Ueda, Y. Takeyama et al., "Significant increase of serum high-mobility group box chromosomal protein 1 levels in patients with severe acute pancreatitis," Pancreas, vol. 33, no. 4, pp. 359-363, 2006.

[37] R. Yang, T. Harada, K. P. Mollen et al., "Anti-HMGB1 neutralizing antibody ameliorates gut barrier dysfunction and improves survival after hemorrhagic shock," Molecular Medicine, vol. 12, no. 4-6, pp. 105-114, 2006.

[38] H. Chen, F. Li, J.-G. Jia, Y.-P. Diao, Z.-X. Li, and J.-B. Sun, "Effects of traditional chinese medicine on intestinal mucosal permeability in early phase of severe acute pancreatitis," Chinese Medical Journal, vol. 123, no. 12, pp. 1537-1542, 2010.

[39] X. Shen and W.-Q. Li, "High-mobility group box 1 protein and its role in severe acute pancreatitis," World Journal of Gastroenterology, vol. 21, no. 5, pp. 1424-1435, 2015.

[40] X. Wang, R. Andersson, V. Soltesz, P. Leveau, and I. Ihse, "Gut origin sepsis, macrophage function, and oxygen extraction associated with acute pancreatitis in the rat," World Journal of Surgery, vol. 20, no. 3, pp. 299-308, 1996.

[41] G. Ramadori, F. Moriconi, I. Malik, and J. Dudas, "Physiology and pathophysiology of liver inflammation, damage and repair," Journal of Physiology and Pharmacology, vol. 59, no. 1, pp. 107117, 2008.

[42] P. F. Lalor and D. H. Adams, "The liver: a model of organspecific lymphocyte recruitment," Expert reviews in molecular medicine, vol. 4, no. 2, pp. 1-16, 2002.

[43] Á. K. Kocsis, A. Szabolcs, P. Hofner et al., "Plasma concentrations of high-mobility group box protein 1 , soluble receptor for advanced glycation end-products and circulating DNA in patients with acute pancreatitis," Pancreatology, vol. 9, no. 4, pp. 383-391, 2009.

[44] L. Schneider, B. Jabrailova, O. Strobel, T. Hackert, and J. Werner, "Inflammatory profiling of early experimental necrotizing pancreatitis," Life Sciences, vol. 126, Article ID 14285, pp. 76-80, 2015.

[45] G. Li, X. Wu, L. Yang et al., "TLR4-mediated NF- $\kappa$ B signaling pathway mediates HMGB1-induced pancreatic injury in mice with severe acute pancreatitis," International Journal of Molecular Medicine, vol. 37, no. 1, pp. 99-107, 2016.

[46] T. Zhang, M. Xia, Q. Zhan, Q. Zhou, G. Lu, and F. An, "Sodium butyrate reduces organ injuries in mice with severe acute 
pancreatitis through inhibiting HMGB1 expression," Digestive Diseases and Sciences, vol. 60, no. 7, pp. 1991-1999, 2015.

[47] A. Tsung, R. Sahai, H. Tanaka et al., "The nuclear factor HMGB1 mediates hepatic injury after murine liver ischemiareperfusion," Journal of Experimental Medicine, vol. 201, no. 7, pp. 1135-1143, 2005.

[48] R. Yang, S. Zhang, A. Cotoia, N. Oksala, S. Zhu, and J. Tenhunen, "High mobility group B1 impairs hepatocyte regeneration in acetaminophen hepatotoxicity," BMC Gastroenterology, vol. 12, article 45, 2012.

[49] D. J. Antoine, D. P. Williams, A. Kipar, H. Laverty, and B. Kevin Park, "Diet restriction inhibits apoptosis and HMGB1 oxidation and promotes inflammatory cell recruitment during acetaminophen hepatotoxicity," Molecular Medicine, vol. 16, no. 11-12, pp. 479-490, 2010.

[50] A. Lau, S. Wang, W. Liu, A. Haig, Z.-X. Zhang, and A. M. Jevnikar, "Glycyrrhizic acid ameliorates HMGB1-mediated cell death and inflammation after renal ischemia reperfusion injury," American Journal of Nephrology, vol. 40, no. 1, pp. 84-95, 2014.

[51] Y.-M. Hu, M.-H. Pai, C.-L. Yeh, Y.-C. Hou, and S.-L. Yeh, "Glutamine administration ameliorates sepsis-induced kidney injury by downregulating the high-mobility group box protein1-mediated pathway in mice," American Journal of PhysiologyRenal Physiology, vol. 302, no. 1, pp. F150-F158, 2012.

[52] H. Sawa, T. Ueda, Y. Takeyama et al., "Blockade of high mobility group box-1 protein attenuates experimental severe acute pancreatitis," World Journal of Gastroenterology, vol. 12, no. 47, pp. 7666-7670, 2006.

[53] R. L. C. Kao, X. Xu, A. Xenocostas et al., "Induction of acute lung inflammation in mice with hemorrhagic shock and resuscitation: role of HMGB1," Journal of Inflammation, vol. 11, no. 1, pp. 30-37, 2014.

[54] M. Entezari, M. Javdan, D. J. Antoine et al., "Inhibition of extracellular HMGB1 attenuates hyperoxia-induced inflammatory acute lung injury," Redox Biology, vol. 2, no. 1, pp. 314-322, 2014.

[55] Z.-G. Luan, X.-J. Zhang, X.-H. Yin et al., "Downregulation of HMGB1 protects against the development of acute lung injury after severe acute pancreatitis," Immunobiology, vol. 218, no. 10, pp. 1261-1270, 2013.

[56] R. Allam, S. V. R. Kumar, M. N. Darisipudi, and H.-J. Anders, "Extracellular histones in tissue injury and inflammation," Journal of Molecular Medicine, vol. 92, no. 5, pp. 465-472, 2014.

[57] J. Xu, X. Zhang, M. Monestier, N. L. Esmon, and C. T. Esmon, "Extracellular histones are mediators of death through TLR2 and TLR4 in mouse fatal liver injury," Journal of Immunology, vol. 187, no. 5, pp. 2626-2631, 2011.

[58] J. Xu, X. Zhang, R. Pelayo et al., "Extracellular histones are major mediators of death in sepsis," Nature Medicine, vol. 15, no. 11, pp. 1318-1321, 2009.

[59] R. Allam, C. R. Scherbaum, M. N. Darisipudi et al., "Histones from dying renal cells aggravate kidney injury via TLR2 and TLR4," Journal of the American Society of Nephrology, vol. 23, no. 8, pp. 1375-1388, 2012.

[60] D. L. Rosin and M. D. Okusa, "Dying cells and extracellular histones in AKI: beyond a NET effect?" Journal of the American Society of Nephrology, vol. 23, no. 8, pp. 1275-1277, 2012.

[61] M. Bosmann, J. J. Grailer, R. Ruemmler et al., "Extracellular histones are essential effectors of C5aR- and C5L2-mediated tissue damage and inflammation in acute lung injury," FASEB Journal, vol. 27, no. 12, pp. 5010-5021, 2013.
[62] S. F. De Meyer, G. L. Suidan, T. A. Fuchs, M. Monestier, and D. D. Wagner, "Extracellular chromatin is an important mediator of ischemic stroke in mice," Arteriosclerosis, Thrombosis, and Vascular Biology, vol. 32, no. 8, pp. 1884-1891, 2012.

[63] S. Hoyos, S. Granell, N. Heredia, O. Bulbena, D. Closa, and L. Fernández-Cruz, "Influence of portal blood on the development of systemic inflammation associated with experimental acute pancreatitis," Surgery, vol. 137, no. 2, pp. 186-191, 2005.

[64] B. Gloor, T. A. Blinman, D. A. Rigberg et al., "Kupffer cell blockade reduces hepatic and systemic cytokine levels and lung injury in hemorrhagic pancreatitis in rats," Pancreas, vol. 21, no. 4, pp. 414-420, 2000.

[65] H.-B. Liu, N.-Q. Cui, D.-H. Li, and C. Chen, "Role of Kupffer cells in acute hemorrhagic necrotizing pancreatitis-associated lung injury of rats," World Journal of Gastroenterology, vol. 12, no. 3, pp. 403-407, 2006.

[66] C. M. Pastor, A. Vonlaufen, F. Georgi, A. Hadengue, P. Morel, and J.-L. Frossard, "Neutrophil depletion-but not prevention of Kupffer cell activation-decreases the severity of ceruleininduced acute pancreatitis," World Journal of Gastroenterology, vol. 12, no. 8, pp. 1219-1224, 2006.

[67] Y. Sailai, X. Yu, P. Baiheti, H. Tang, Y. Li, and M. Xu, "Influence of nuclear factor $\kappa \mathrm{b}$ activation on inflammatory mediators of alveolar macrophages in rats with acute necrotizing pancreatitis," Journal of Investigative Medicine, vol. 58, no. 1, pp. 38-42, 2010.

[68] L. Schneider, T. Hackert, T. Longerich et al., "Effects of gadolinium chloride and glycine on hepatic and pancreatic tissue damage in alcoholic pancreatitis," Pancreas, vol. 39, no. 4, pp. 502-509, 2010.

[69] L. Ulloa, M. Ochani, H. Yang et al., "Ethyl pyruvate prevents lethality in mice with established lethal sepsis and systemic inflammation," Proceedings of the National Academy of Sciences of the United States of America, vol. 99, no. 19, pp. 12351-12356, 2002. 


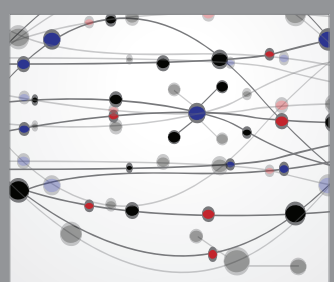

The Scientific World Journal
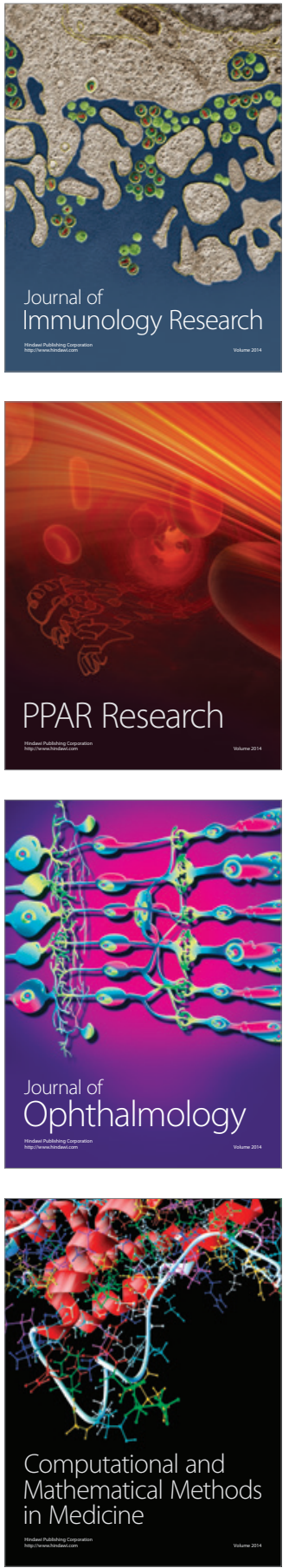

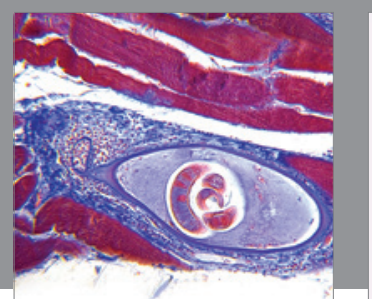

Gastroenterology Research and Practice
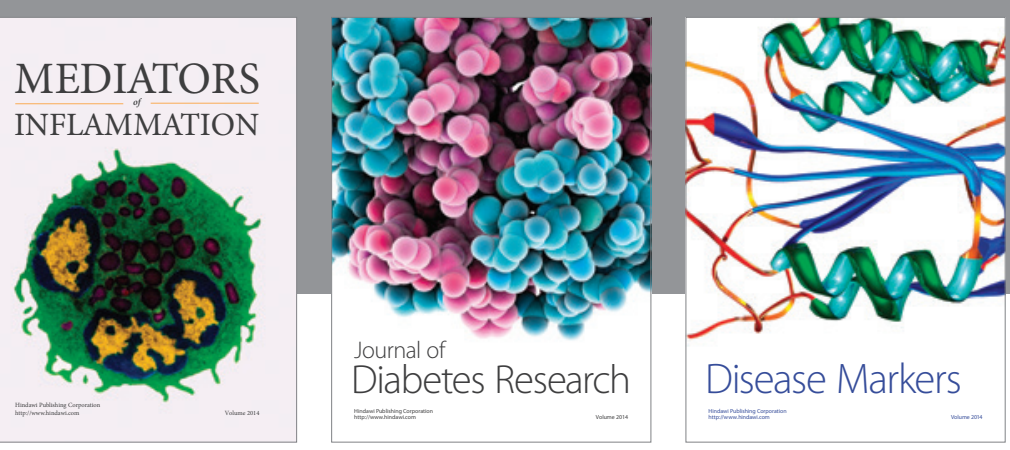

Disease Markers

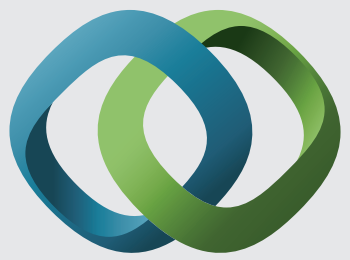

\section{Hindawi}

Submit your manuscripts at

https://www.hindawi.com
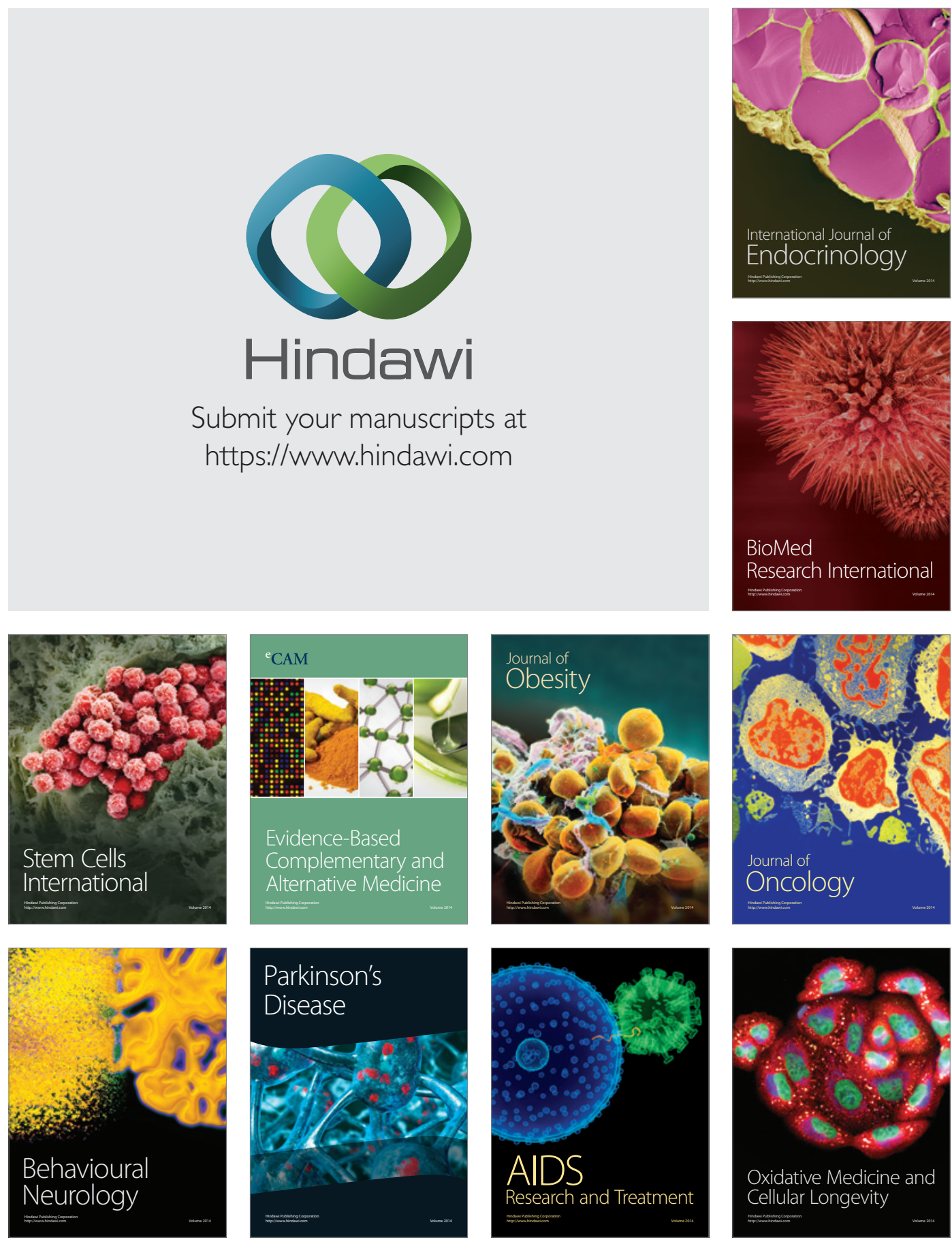